\title{
Saturation of deuterium retention in self-damaged tungsten exposed to high-flux plasmas.
}

\author{
M H J 't Hoen ${ }^{1}$, B Tyburska-Püschel ${ }^{2,3}$, K Ertl $^{3}$, M Mayer ${ }^{3}$, J Rapp ${ }^{1}$, A W Kleyn ${ }^{1,4}$, \\ P A Zeijlmans van Emmichoven ${ }^{1}$ \\ ${ }^{1}$ FOM Institute for Plasma Physics Rijnhuizen, NL-3439 MN Nieuwegein, The Netherlands \\ ${ }^{2}$ Department of Engineering Physics, University of Wisconsin-Madison, Madison, WI, U.S.A \\ ${ }^{3}$ Max-Planck-Institut für Plasmaphysik, Boltzmannstraße 2, D-85748 Garching, Germany \\ ${ }^{4}$ University of Amsterdam, Science Park 904, NL-1098 XH Amsterdam, The Netherlands
}

\begin{abstract}
Polycrystalline, annealed tungsten targets were bombarded with $12.3 \mathrm{MeV} \mathrm{W}^{4+}$ ions to various damage levels. Deuterium was implanted by high-flux plasmas in Pilot-PSI $\left(>10^{24} \mathrm{~m}^{-2} \mathrm{~s}^{-1}\right)$ at a surface temperature below $525 \mathrm{~K}$. Deuterium retention has been studied by Nuclear Reaction Analysis and by Thermal Desorption Spectroscopy. We found that deuterium retention is strongly enhanced by the tungsten bombardment and that saturation occurs at a $\mathrm{W}^{4+}$ fluence of about $3 \cdot 10^{17} \mathrm{~m}^{-2}$. The maximum deuterium concentration in the damaged region was measured to be 1.4 at.\%. This is in accordance with other experiments that were carried out at much lower fluxes. We therefore conclude that the saturation behaviour and the maximum retention are not affected by the high fluxes used in our experiments.
\end{abstract}

A simple geometric model is presented that assumes that the saturation solely originates in the tungsten irradiation and that explains it in terms of overlapping saturated volumes. The saturated volume per incident $\mathrm{MeV}$ ion amounts to $3 \cdot 10^{4} \mathrm{~nm}^{3}$. From our results, we are able to obtain an approximate value for the average occupation number of the vacancies.

\section{Introduction}

In future magnetic fusion devices as ITER, tungsten is foreseen as divertor material. The divertor has to withstand high density, low temperature plasma fluxes. Good thermal properties as its high melting point, high thermal conductivity and low erosion rate make tungsten favourable over other materials. However, there is concern that hydrogen isotopes are retained in tungsten. Firstly, for safety reasons, it has been decided for ITER that the tritium inventory should be kept below $700 \mathrm{~g}$ [1]. Secondly, for efficiency reasons, it is important that the fuel is not retained in the wall. Understanding the retention properties of $\mathrm{W}$ is important for ITER and for fusion reactors beyond ITER. Continuous bombardment with $14.1 \mathrm{MeV}$ neutrons degrades material properties and introduces damage into the material. In this paper we focus on the influence of damage in tungsten on deuterium retention.

Several studies have shown that irradiation damage strongly enhances deuterium retention [2] [3] [4]. Tyburska et al [2] noticed that a linear increase of damage did not lead to a linear increase of deuterium stored in the sample and that the defects can be removed by annealing. Saturation of the deuterium retention was observed at 0.3 displacements per atom (dpa), independent of implantation fluence. Wright et al [3], who did similar experiments at a much higher flux, showed that the deuterium retained in damaged tungsten saturates below 0.22 dpa. 
The main aim of this investigation is to determine the irradiation damage level where deuterium retention in tungsten saturates for high-flux plasmas, and to understand the saturation behaviour. A systematic study of deuterium retention in tungsten as a function of target temperature and irradiation damage level was performed. Analysis was done by Nuclear Reaction Analysis and Thermal Desorption Spectroscopy. The results are explained by a simple geometric model based on overlapping volumes.

\section{Experiment}

\subsection{Target preparation}

Polycrystalline tungsten targets (PLANSEE, $99.96 \%$ purity) were annealed for 1 hour at $1273 \mathrm{~K}$ at a background pressure of $5 \cdot 10^{-4} \mathrm{~Pa}$. The dimensions of the targets are $20 \mathrm{~mm}$ in diameter and $1 \mathrm{~mm}$ in thickness. In the present experiments, unpolished targets were used. The cleanliness of the targets was checked by XPS before and after plasma exposure. In both cases, the XPS signal was dominated by carbon (40-70 \%) and oxygen (20-40\%). This can be ascribed to native carbon and oxide layers of a few nanometres thick, present on the targets due to their exposure to air. At ambient conditions hydrocarbons are deposited onto the tungsten target. During plasma exposure, the chemical erosion of carbon [5] is an efficient process and formation of water removes the oxide layer. It is therefore likely that during plasma exposures no carbon nor oxide was present. The tungsten signal was only about $10 \%$. This low signal is partly caused by the rough surface of the target; polished samples showed an increase of the signal by a factor 3. With XPS, trace amounts of copper and calcium were measured after Pilot-PSI [6] exposure. They were deposited on the targets during plasma exposure. Their contribution to the XPS signal was very limited and well below $1 \%$.

\section{$2.2 \mathrm{~W}^{4+}$ irradiation}

Damage caused by neutron irradiation was simulated by heavy ion bombardment. Radiation damage by neutrons and by heavy ions is to some extent comparable [7]. For both neutrons and heavy ions, the ratio of low-energy to high-energy primary knock-on atoms is low, and they both create dense collision cascades [8]. However, one should realize that there are also differences between heavy ion and neutron irradiation. Damage created by heavy ions is concentrated in a narrow region below the surface, while neutron damage exhibits a homogeneous defect concentration very deep in the material. Furthermore, the detailed nature of the defects is unclear and may be different and neutron collisions will lead to transmutations whereas heavy ions will not.

The samples were bombarded at normal incidence with $12.3 \mathrm{MeV} \mathrm{W}$ ions. The $\mathrm{W}^{4+}$ ions, accelerated through a potential difference of $3.075 \mathrm{MV}$, were implanted with the $3 \mathrm{MV}$ tandem ion accelerator at IPPGarching. In order to obtain homogeneous irradiation, the beam $(\varnothing \sim 3 \mathrm{~mm})$ was raster scanned across the surface. The area damaged by the tungsten bombardment was $12 \mathrm{~mm}$ in diameter. The damage rate at which the targets were damaged was $(3+/-1) \cdot 10^{-4} \mathrm{dpa} \mathrm{s}^{-1}$. During the tungsten irradiation, the targets were kept at room temperature. To predict the irradiation damage, calculations were done with the binary collision transport code SRIM . SRIM profiles were calculated for $0 \mathrm{~K}$. The absolute level of displacement damage decreases approximately linearly with an increase of the displacement threshold energy $\left(E_{d}\right)$. In literature, a wide range of values has been used: Sakamoto et al [9] and Tokunaga et al [10] used $40 \mathrm{eV}$

\footnotetext{
${ }^{\text {a }}$ www.srim.org
} 
determined by Lucasson et al [11]. Maury et al [12] measured by electron irradiation a value of $42 \pm 1 \mathrm{eV}$ in the $<100>$ direction and $44 \pm 1 \mathrm{eV}$ for $<111>$ orientation. Xu et al [13] showed with molecular dynamics simulations that the displacement threshold energy is indeed strongly dependent on the crystal orientation and ranges from $68 \mathrm{eV}$ up to $250 \mathrm{eV}$. Others, like Ogorodnikova et al [14], use an average displacement energy of $90 \mathrm{eV}$ as reported by [15]. In this article the average value of $90 \mathrm{eV}$ is used, unless stated otherwise.

The SRIM results are shown in figure 1. Damage profiles extend to $1.5 \mu \mathrm{m}$ below the surface and have a maximum damage level at about $0.8 \mu \mathrm{m}$ depth. The targets were subjected to $\mathrm{W}^{4+}$ ion fluences up to $6.5 \cdot 10^{17} \mathrm{~m}^{-2}$, which corresponds to peak damage levels of up to $0.45 \mathrm{dpa}$. These peak damage levels are used as reference for our experiments. For comparison to the results of Tyburska et al, the 0.4 dpa damage profile created by $5.5 \mathrm{MeV} \mathrm{W}^{+}$ions is also depicted. It is clear that $12.3 \mathrm{MeV}$ ions penetrate deeper in the material and that the maximum damage level occurs at larger depth.

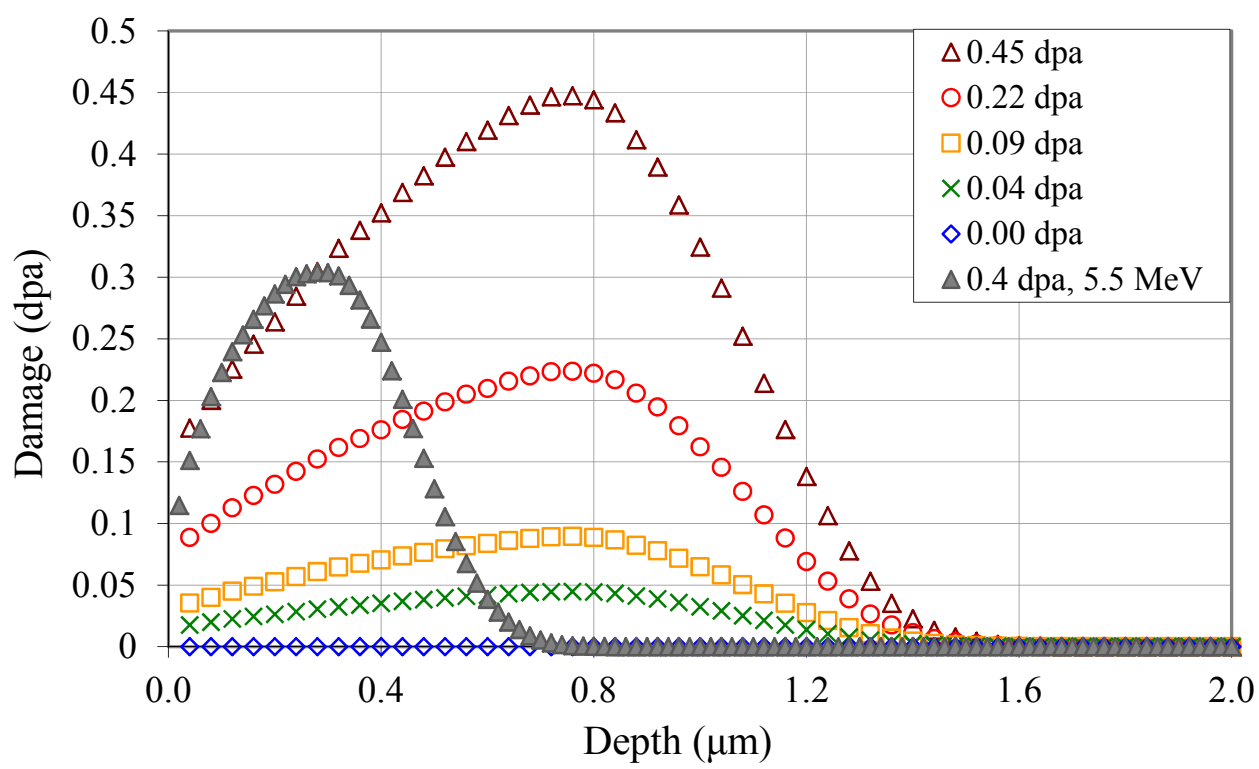

Figure 1: SRIM results of damage distribution for tungsten irradiated by $12.3 \mathrm{MeV} \mathrm{W}^{4+}$ ions. For comparison to the results of Tyburska et al [2], the 0.3 dpa damage profile created by $5.5 \mathrm{MeV} \mathrm{W}^{+}$ions is depicted as well (reconstructed from [2] and scaled to $E_{d}=90 \mathrm{eV}$ ).

\subsection{Deuterium implantation by plasma exposure}

Pilot-PSI is a linear plasma generator for plasma surface interaction (PSI) studies in support of the international fusion experiment ITER [6] [16]. Deuterium plasma is produced by a cascaded arc, which facilitates the production of unique plasma conditions. Particle fluxes of up to $10^{24} \mathrm{~m}^{-2} \mathrm{~s}^{-1}$ are obtained with energy fluxes up to $10 \mathrm{MW} / \mathrm{m}^{2}$ and electron temperatures of $0.5-5 \mathrm{eV}$. The damaged targets have been exposed for $100 \mathrm{~s}$ in Pilot-PSI in an axial magnetic field of $0.4 \mathrm{~T}$.

Electron density and temperature of the plasma beam were determined by the Thomson scattering technique [17]. The plasma beam has a Gaussian profile with an electron density of about $3.0 \cdot 10^{20} \mathrm{~m}^{-3}$ in the centre and a full width half maximum of $\sim 1 \mathrm{~cm}$. The maximum electron temperature was measured to be $0.7 \mathrm{eV}$. 
During plasma exposure, the targets were electrically floating. Assuming that the ion temperature equals the electron temperature and using the Bohm criterion [18], the peak deuterium ion plasma flux can be determined to be $1.2 \cdot 10^{24} \mathrm{~m}^{-2} \mathrm{~s}^{-1}$. The flux on and the surface temperature of the target are shown in figure 2. The deuterium implantation area $(\varnothing 16 \mathrm{~mm})$ covered the pre-irradiated area. The surface temperature is determined by the heat flux of the plasma and by cooling of the target. In all experiments, temperature profiles of the surface of the target have been measured with a fast infrared camera (FLIR SC7500-MB). The maximum surface temperature was $525 \mathrm{~K}+/-10 \mathrm{~K}$. The emissivity of tungsten at these temperatures is very low and dependent of the surface structure. It is therefore possible that a systematic error of maximum $30 \mathrm{~K}$ was introduced by an error in emissivity coefficient. The cooling of the target was very effective; after switching off the plasma, the temperature of the targets was back to room temperature within typically $1-2$ seconds.

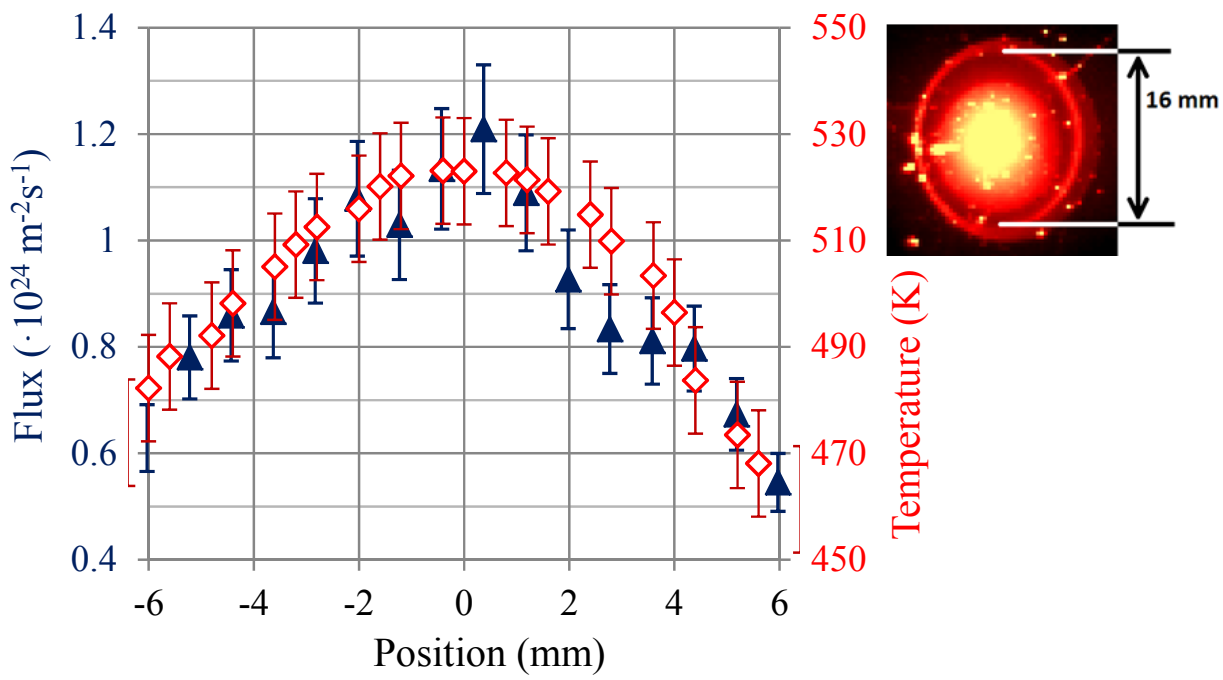

Figure 2: Radial dependence of the plasma flux (closed triangles), and surface temperature (open diamonds). The insert shows a typical infrared image.

\subsection{Nuclear Reaction Analysis}

Nuclear Reaction Analysis (NRA) of the targets was performed at IPP-Garching four weeks after plasma exposure. The nuclear reaction $\mathrm{D}\left({ }^{3} \mathrm{He}, \mathrm{p}\right)^{4} \mathrm{He}$ was used to obtain a local measurement of the deuterium concentration as function of depth [19] [20]. The ${ }^{3} \mathrm{He}$ beam spot of $1 \mathrm{~mm}$ in diameter was positioned at four spots on the target to extract a radial scan. At each position, the energy was scanned from $690 \mathrm{keV}$ to $4.0 \mathrm{MeV}$ to determine the deuterium concentration with a resolution of $\sim 0.5 \mathrm{~m}$ down to $6 \mathrm{~m}$ depth. The depth profiles of the retained deuterium were calculated from the measured proton energy distributions by use of the SimNRA program [21]. The surface roughness of the targets did not allow us to extract information from the alpha spectrum to obtain a near surface $\mathrm{D}$ concentration with a better resolution. The total amount of deuterium present in the top $6 \mathrm{~m}$ of the target was obtained by integrating the depth profiles.

\subsection{Thermal Desorption Spectroscopy}

The targets were analysed with Thermal Desorption Spectroscopy (TDS) at FOM-Rijnhuizen seven weeks after plasma exposure. The tungsten target was clamped to a ceramic heater and heated with a linear 
temperature ramp of $1 \mathrm{~K} / \mathrm{s}$ to $1273 \mathrm{~K}$. A Balzers QMA125 Quadrupole Mass Spectrometer (QMS) monitored the mass $4\left(\mathrm{D}_{2}\right)$ and mass 3 (HD) signals in the residual gas in the chamber, to determine the total amount of deuterium released from the target during the temperature ramp. The absolute sensitivity was determined using calibrated leaks of $\mathrm{H}_{2}$ and $\mathrm{D}_{2}$. For the sensitivity of the mass 3 signal, the average of the sensitivities of mass 2 and mass 4 was taken.

\section{Results}

\subsection{Nuclear Reaction Analysis}

NRA was carried out at several positions on the target to obtain deuterium depth distributions as function of temperature and flux. The results are shown in figure 3a-d. Also the retention for an undamaged target is shown. From figure 3, it is clear that $\mathrm{W}^{4+}$ pre-irradiation strongly enhances deuterium retention. The deuterium retention decreases as function of depth. The deuterium retention beyond $3 \mu \mathrm{m}$ was below the detection limit of $10 \mathrm{ppm}$.
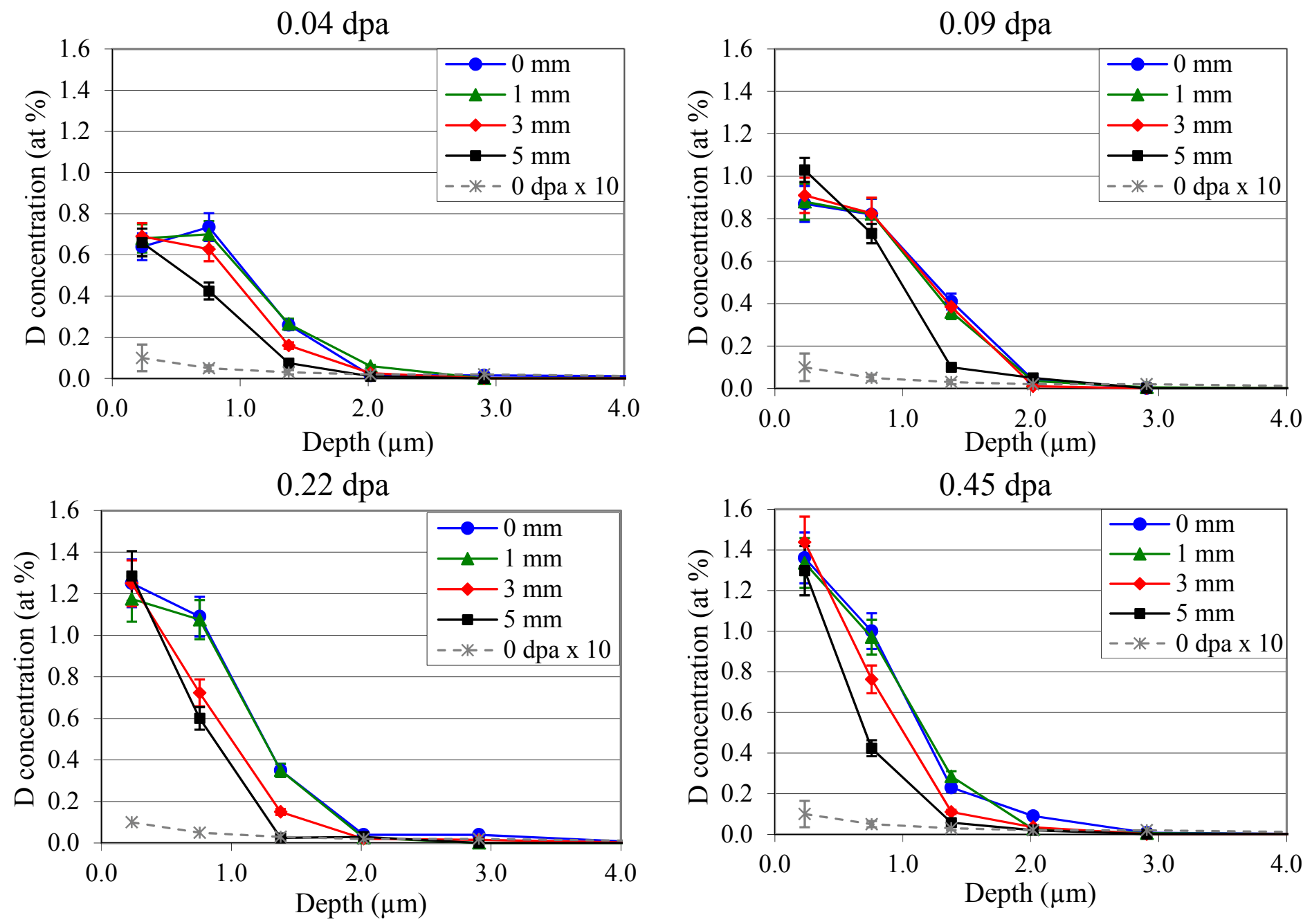

Figure 3: Depth distributions of retained deuterium in damaged tungsten targets, pre-irradiated to respectively (a) 0.045 , (b) 0.09 , (c) 0.22 and (d) 0.45 dpa. The shown D depth profiles were measured at 4 
spots on the target: in the centre $(\sim 525 \mathrm{~K}) ; 1 \mathrm{~mm}$ off centre $(\sim 520 \mathrm{~K}) ; 3 \mathrm{~mm}$ off centre $(\sim 510 \mathrm{~K}) ; 5 \mathrm{~mm}$ off centre $(\sim 480 \mathrm{~K})$. The grey data points and dotted line indicates the deuterium retention of an undamaged tungsten target (multiplied by ten).

At the measurement points in the centre of the target and $1 \mathrm{~mm}$ off center, which were exposed to the highest particle flux and which had the highest surface temperature, the deuterium was found deepest in the material. This agrees with the observations of Wright et al [3], who found that in this temperature regime, deuterium retention is primarily determined by diffusion: at the highest surface temperature, deuterium diffuses deepest into the tungsten material (figure 3).

For the measurements nearest to the surface $(0.25 \mu \mathrm{m})$, deuterium retention is independent of position and thus of surface temperature and particle flux. Deuterium retention does clearly increase with damage level, which indicates that the retention at the surface is mostly determined by the defect concentration. The maximum in deuterium retention is about 1.4 at.\%, which agrees well with the maximum deuterium concentration of 1.5 at.\% found by others [2] [3] [22]. Both Tyburska et al [2] and Fukumoto et al [22] found an additional surface effect, where the retained deuterium is much higher in a very thin layer at the surface. By investigation of the surface morphology, it became clear that these high values originated from cracks and blister formation. This surface effect can also be observed in our experiments, as is clear from the experiments on the undamaged target, but only gives a minor contribution to the retention of the damaged targets (caused by the limited depth resolution of $0.5 \mu \mathrm{m}$ ).

The measured deuterium retention profiles do not match the calculated damage profiles as shown in figure 1 , with the measured retention deeper in the material being significantly lower than expected from the damage profiles. This also holds when the effect of saturation on the damage profiles is considered, leading to flatter profiles. The mismatch is probably caused by the limited diffusion. This is supported by the fact that for a specific target, the retention at the surface is the same for all positions whereas deeper in the material retention is larger in the center (where flux and temperature are higher).

The integrated NRA data, i.e. the deuterium retained in the first $6 \mu \mathrm{m}$ of the target, is shown in figure 4 . Clearly, the retention is close to its saturation value at a $\mathrm{W}^{4+}$ pre-irradiation fluence of $3 \cdot 10^{17} \mathrm{~m}^{-2}$. Using a value of $90 \mathrm{eV}$ for the displacement energy, this corresponds to $0.2 \mathrm{dpa}$. 


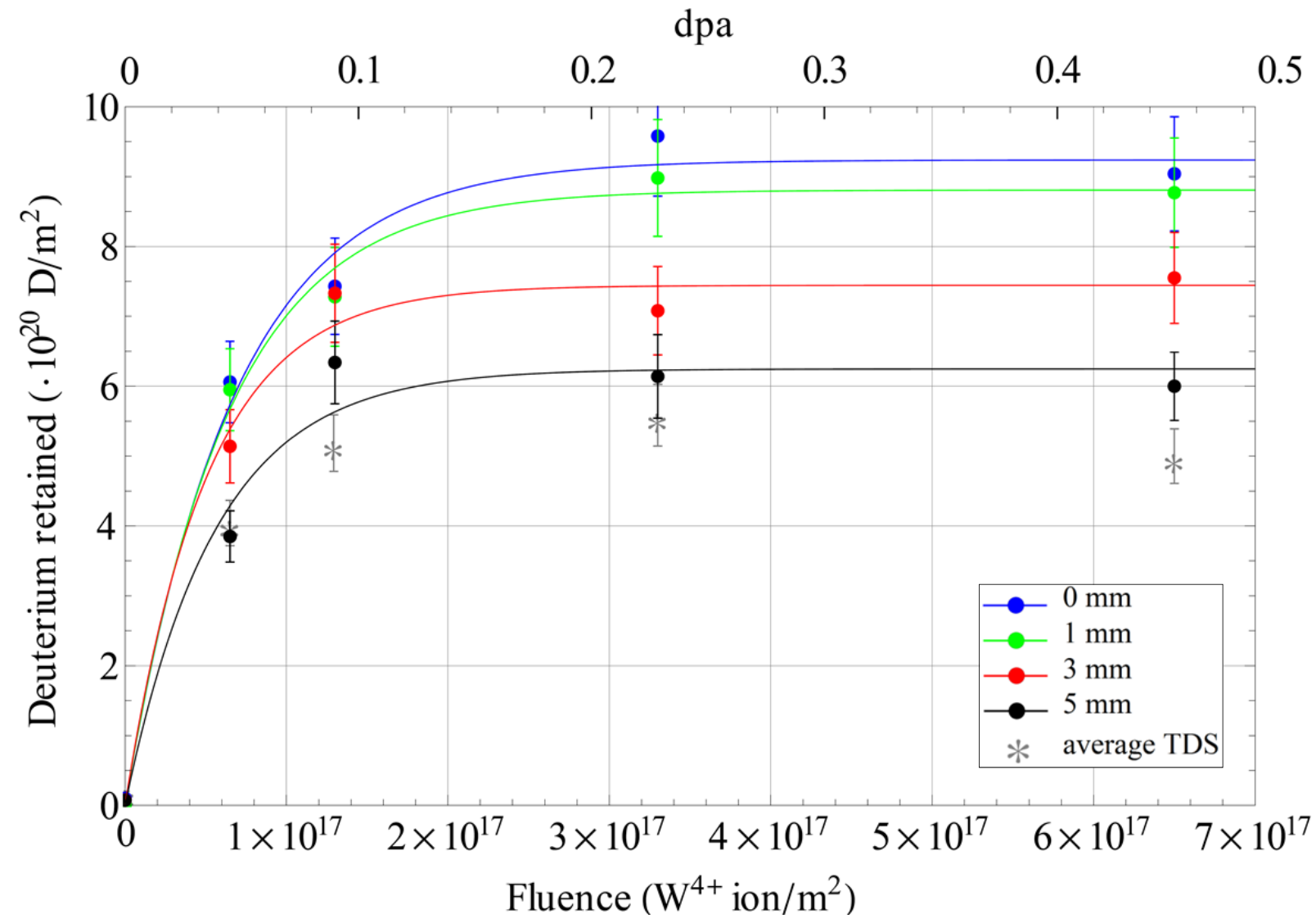

Figure 4: Amount of deuterium retained in the first $6 \mathrm{~m}$ as a function of pre-irradiation $\mathrm{W}$ ion fluence. Deuterium retention is significantly enhanced by $\mathrm{W}^{4+}$ pre-irradiation and increases with pre-irradiation damage. Saturation of deuterium retention is reached around $0.2 \mathrm{dpa}$. A comparison with the TDS data is made by dividing the total amount of deuterium desorbed during TDS by the surface area.

\subsection{Thermal Desorption Spectroscopy}

We investigated our targets with TDS to get information on the defect types in which the deuterium is trapped. The TDS results for the $\mathrm{D}_{2}$ signal are shown in figure 5. The undamaged target shows a small low temperature peak at around $550 \mathrm{~K}$, caused by deuterium trapped in intrinsic defects usually associated with dislocations and grain boundaries [3]. It is likely that these naturally exist in the material, as the annealing temperature of $1273 \mathrm{~K}$ is still below the recrystallization temperature of tungsten. Since the deuterium ion energy is much lower than the displacement threshold of tungsten, it does not seem likely that these defects are created by plasma exposure. However, several mechanisms have been suggested for defect creation by deuterium ion implantation below this threshold, e.g.: plastic deformation by deuterium super-saturation [23] and creation of vacancies by recoil implantation of carbon and oxygen impurities arriving at the tungsten surface [24].

Pre-irradiation of the targets enhances the intensity of the low temperature peak and introduces a high temperature peak at about $850 \mathrm{~K}$. The enhancement and broadening of the low temperature peak towards higher temperatures can be explained by defect creation. In literature, various defect types have been identified in the temperature range 400-650K, i.e., dislocations and grain boundaries [25] [26], and 
single vacancies (with one [26] [27] or two D atoms trapped [28]). The high temperature peak is usually associated with deuterium trapped in vacancy clusters [22] [25] [29]. The high temperature peak measured by Wright et al [3] is significantly less pronounced than the one in our experiments. This might be caused by the lower surface temperature during plasma exposure as used in their experiments (480 K), which limits the clustering of vacancies.

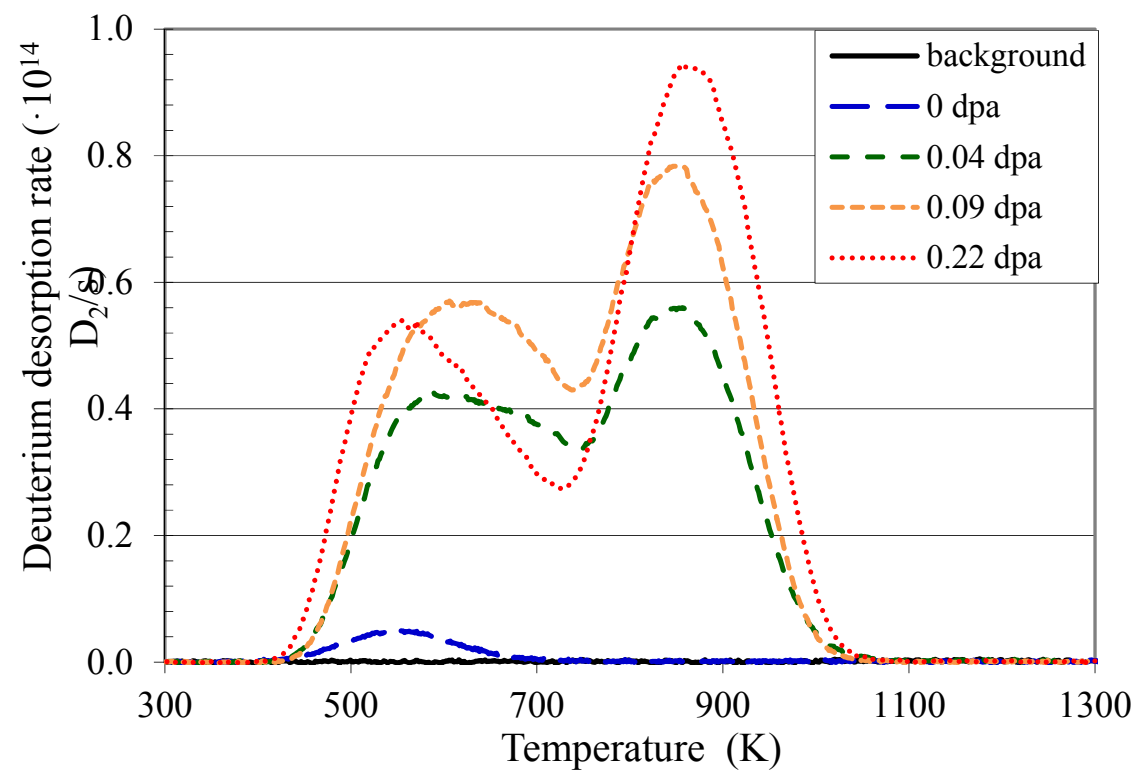

Figure 5: Thermal desorption spectra $\left(\mathrm{D}_{2}\right.$ signal $)$ of the targets, temperature ramp was $1 \mathrm{~K} / \mathrm{s}$. The background signal was measured at a target that was not irradiated by tungsten ions, nor exposed to deuterium plasma, and is virtually 0 at all temperatures. Unfortunately, the measurement at 0.45 dpa was not successful due to technical problems in the heating rate. However, the integrated TDS profile could be used to calculate the total deuterium retention.

The total deuterium retained by TDS divided by the damaged surface area (Ø $12 \mathrm{~mm})$ is shown in figure 4. The average value obtained by TDS is somewhat lower than the local D retention (NRA) at $5 \mathrm{~mm}$ offcenter. This may be caused by a misalignment between the center of the plasma beam and the center of the self-implantation area of $1 \mathrm{~mm}$.

\section{Model}

The deuterium retention in the first $6 \mathrm{~m}$ underneath the surface as function of pre-irradiation fluence is shown in figure 4 . The retention clearly saturates at a $\mathrm{W}^{4+}$ fluence of about $3 \cdot 10^{17} \mathrm{~m}^{-2}$. We developed a simple geometric model that assumes that the saturation solely originates in the tungsten pre-irradiation and that explains this saturation in terms of overlapping volumes.

The model is an extension of a 2D model, where a surface with area $A$ is randomly covered with objects with surface area $a$. The expectation value of the fraction $f(n)$ of surface $A$ covered with $n$ such objects is given by the following differential equation:

$\frac{d f}{d n}=\frac{a}{A}[1-f(n)]$ 
The solution of this equation is given by:

$f(n)=1-\exp (-n a / A)$

$\mathrm{MeV}$ ions bombarding a material create Frenkel pairs. At sufficiently high pre-irradiation fluence, the material becomes saturated with Frenkel pairs: a new vacancy created will immediately recombine with an interstitial present in the material. This spontaneous recombination leads to a maximum concentration of vacancies. Each single vacancy occupies an average spontaneous recombination volume, $V^{\mathrm{vac}}$.

Each individual $\mathrm{MeV}$ ion creates a unique, very complex, 3D distribution of vacancies. SRIM simulates these individual distributions and calculates, among others, the number of vacancies as function of depth averaged over many $\mathrm{MeV}$ ions. The shape of this average vacancy distribution as function of depth $d$, $\operatorname{vac}_{\text {SRIM }}(d)$, resembles the graphs shown in figure 1. In our model we assume that, instead of a unique distribution, each individual $\mathrm{MeV}$ ion creates this average vacancy distribution. The average effective damage volume of an ion, $V_{\mathrm{eff}}^{\text {ion }}$, can be expressed in terms of $V^{\mathrm{vac}}$ in the following way:

$V_{\mathrm{eff}}^{\mathrm{ion}}=V^{\mathrm{vac}} \cdot \sum_{\text {layers }} v^{\mathrm{a}} c_{\mathrm{SRIM}}(d)$

$V_{\text {eff }}^{\text {ion }}$ represents the damage volume created by a single $\mathrm{MeV}$ ion saturated with vacancies. With the $\mathrm{MeV}$ ions incident at random positions on the target, the damage volumes will start to overlap as soon as the fluence is sufficiently high, and saturation occurs.

We extended the 2D case (equation 2) to a 3D model by splitting up the target in $N$ layers, parallel to the surface and of equal thickness. The surface area $a$ in equation 2 has to be taken different for each layer. The relative values are determined by the shape of $\operatorname{vac}_{\text {SRIM }}(d)$. We furthermore replace the fraction $f(n)$ by the deuterium retention. The model leads to the following function, where the summation is taken over the $N$ contributing layers:

$D_{\text {ret }}(F)=\frac{D_{\text {sat }}}{N} \sum_{\text {layers }}\left\{1-\exp \left[-F \cdot \operatorname{vac}_{\mathrm{SRIM}}(d) \cdot V^{\mathrm{vac}} / \Delta d\right]\right\}$

Here, $D_{\text {ret }}(F)$ is the deuterium retention as function of the pre-irradiation fluence $F$ and $D_{\text {sat }}$ the maximum deuterium retention when the sample is saturated with vacancies. The thickness of the layers is given by $\Delta d$. The function (equation 4) was fitted to the four sets of data points shown in figure 4 with $D_{\text {sat }}$ and $V^{\mathrm{vac}}$ as fit parameters. Note that, although $\operatorname{vac}_{\mathrm{SRIM}}(d)$ and $V^{\mathrm{vac}}$ are both directly affected by the choice of $\mathrm{E}_{\mathrm{d}}, V_{\mathrm{eff}}^{\text {ion }}$ is not.

In figure 4, the data with the fits are shown. It is clear that the fit function describe the measurements quite well. The deuterium retention from a single $\mathrm{MeV}$ ion, incident on a non-irradiated target, amounts to 1.3$1.7 \cdot 10^{4} \mathrm{D} /$ ion, which can be directly obtained from the slope at 0 fluence. The average effective damage volume of an $\mathrm{MeV}$ ion, $V_{\mathrm{eff}}^{\text {ion }}$, was found to be $(3+/-1) \cdot 10^{4} \mathrm{~nm}^{3}$.

\section{Discussion}


We have studied deuterium retention for targets pre-irradiated with $12.3 \mathrm{MeV} \mathrm{W}^{4+}$ ions and subsequently exposed in Pilot-PSI to high-flux plasmas. From integrated NRA data, it is clear that saturation of deuterium retention takes place at a $\mathrm{W}^{4+}$ fluence of about $3 \cdot 10^{17}$ ions $\mathrm{m}^{-2}$, which corresponds to $\sim 0.2 \mathrm{dpa}$. Since the absolute value of the created damage is inversely proportional to the displacement threshold energy, we can rescale the saturation point found by Tyburska et al [2] to $\sim 0.3 \mathrm{dpa}\left(\mathrm{E}_{\mathrm{d}}=90 \mathrm{eV}\right)$, measured at fluxes of about $10^{22} \mathrm{~m}^{-2} \mathrm{~s}^{-1}$. Since these values are very close and, in addition, since the maximum retention levels are quite similar, we conclude that the high flux of $>10^{24} \mathrm{~m}^{-2} \mathrm{~s}^{-1}$ as used in the present experiments does not significantly affect deuterium retention. This agrees with our model that assumes that the saturation behaviour solely originates in the damage created before the $\mathrm{D}$ irradiation.

From the analysis of our measurements, we were able to obtain the average effective damage volume of the $\mathrm{MeV}$ ions used in our experiments and the number of deuterium atoms retained per $\mathrm{MeV}$ ion. With this information an estimate of the occupation number of the vacancies can be made.

We have estimated the number of vacancies created by an $\mathrm{MeV}$ ion in two different ways. The first method uses the number of Frenkel pairs derived from the Norgett-Robinson-Torrens (NRT) formula [30]. The integrated damage energy output files of SRIM were used to calculate the number of Frenkel pairs created by each $\mathrm{MeV}$ ion: on average $2.0 \cdot 10^{4}$ vacancies. Note that the calculated number of vacancies depends on the choice made for $E_{d}$. However, the actual number of vacancies per incident ion is lower, since the NRT formula does not take into account the recombination of defects produced in the collision cascade itself. Molecular Dynamics simulations (MD) have shown that the recombination drastically decreases the amount of vacancies [31] [32]. Troev et al [32] used molecular dynamics simulations of tungsten to determine the cascade efficiency, defined as the ratio between Frenkel pairs calculated by MD and the theoretical number of Frenkel pairs derived by the NRT formula. They found that $20-25 \%$ of the Frenkel pairs survive, the rest annihilates. The damaged volume of the $\mathrm{MeV}$ ion is then left with $4-5 \cdot 10^{3}$ vacancies. The second method uses the spontaneous recombination volume of Frenkel pairs. From our experiments we found an average effective damage volume of an $\mathrm{MeV}$ ion of about $3 \cdot 10^{4} \mathrm{~nm}^{3}$. In literature, the spontaneous recombination volume of Frenkel pairs in tungsten is reported to be in the range of 100-200 at. vol [33] [34] or 1.6-3.2 $\mathrm{nm}^{3}$. Hence, the effective damage volume corresponds to (0.91.9) $\cdot 10^{4}$ vacancies. The results from the two methods are somewhat different, and further investigation is needed.

From our experiments, we deduced a number for the amount of retained deuterium: (1.3-1.7) $10^{4}$ deuterium atoms per incident $\mathrm{MeV}$ ion. Dividing this experimental number by the number of vacancies as discussed above, yields a value for the average occupation number of a vacancy of 2.6-4.2 for method 1 and 0.7-1.8 for method 2. It seems that even though the fluence as used in our experiments was not sufficiently high to fully saturate all created vacancies (diffusion limited, see discussion figure 3), the average occupation number is at least close to 1 . At longer exposure times, therefore, deuterium retention will be enhanced and the average occupation number is anticipated to increase further.

\section{Conclusions}

Polycrystalline, annealed and pre-damaged $\mathrm{W}$ targets were exposed to high-flux plasmas in Pilot-PSI at a surface temperature below $525 \mathrm{~K}$. The deuterium retention has been investigated by NRA and TDS. We found that the deuterium retention is enhanced by pre-irradiation with $12.3 \mathrm{MeV} \mathrm{W}$ ions and that it 
saturates at W ion fluence of $\sim 3 \cdot 10^{17} \mathrm{~m}^{-2}$, which corresponds to $\sim 0.2 \mathrm{dpa}\left(\mathrm{E}_{\mathrm{d}}=90 \mathrm{eV}\right)$. We found that the deuterium saturation retention in the damaged $\mathrm{W}$ is not significantly affected by the high fluxes of $\sim 10^{24}$ $\mathrm{m}^{-2} \mathrm{~s}^{-1}$ used in our experiments, and that it is dominated by the pre-irradiation damage. This is in accordance with the fact that our data fit quite well to the simple geometric model, which assumes that the saturation of deuterium retention in the tungsten material solely originates in the $\mathrm{W}^{4+}$ self-implantation. We further found that the average occupation number of the vacancies is at least close to 1 and that it will increase for higher fluencies.

We used heavy ions $\left(\mathrm{W}^{4+}\right)$ to simulate the effect of neutron irradiation with heavy ion bombardment on tungsten as present in the divertor of fusion reactors like ITER. Assuming neutron damage to be comparable to damage from heavy ions, the hydrogenic retention would amount to a maximum value of 1.4 at. $\%$ for surface temperatures below $525 \mathrm{~K}$. At higher surface temperatures the retention is expected to be less, because of annealing of the vacancies.

In the present study, we focussed on deuterium retention in the first few micrometers. Here, the deuterium retention is dominated by the pre-irradiation damage. Further investigations are needed on the effect of high flux plasmas on surface morphology, blistering etc. These studies will be carried out in the future.

\section{Acknowledgements}

This work, supported by the European Communities under the contract of Association between EURATOM/FOM, was carried out within the framework of the European Fusion Programme with financial support from NWO. The views and opinions expressed herein do not necessarily reflect those of the European Commission.

The authors would like to thank R.S. Al for the technical assistance at the Pilot-PSI measurements, J. Dorner and M. Fußeder (IPP-Garching) for their technical assistance with the ${ }^{3} \mathrm{He}$ beam analysis and

E. Zoethout for the XPS analysis of the samples.

\section{References}

[1] J Roth et al, Recent analysis of key plasma wall interactions issues for ITER, Journal of Nuclear Materials 390-391 (2009) 1-9.

[2] B Tyburska, V Kh Alimov, O V Ogorodnikova, K Schmidt and K Ertl, Deuterium retention in self-damaged tungsten, Journal of Nuclear Materials 395 (2009) 150-155.

[3] G M Wright, M Mayer, K Ertl, G de Saint-Aubin and J Rapp, Hydrogenic retention in irradiated tungsten exposed to high-flux plasma, Nuclear Fusion 50 (2010) 075006.

[4] W R Wampler and R P Doerner, The influence of displacement damage on deuterium retention in tungsten exposed to plasma, Nuclear Fusion 49 (2009) 115023.

[5] J Roth et al, Flux dependence of carbon erosion and implication for ITER, Journal of Nuclear Materials 337-339 (2005) 970-974.

[6] G J van Rooij, V P Veremiyenko, W J Goedheer, B de Groot, A W Kleyn, P H Smeets, T W Versloot, D G Whyte, R Engeln, D C Schram, and N J Lopes Cardozo, Extreme hydrogen plasma densities achieved in a linear plasma generator, Applied Physics Letters 90 (2007) 121501 . 
[7] Gary S. Was, Fundamentals of radiation material science: Metals and Alloys, Springer-Verlag Berlin Heidelberg, New York (2007).

[8] P Vladimirov and S Bouffard, Displacement damage and transmutations in metals under neutron and proton irradiation, Comptes Rendus Physique 9 (2008) 303.

[9] R Sakamoto, T Muroga and N Yoshida, Microstructural evolution induced by low energy hydrogen ion irradiation in tungsten, Journal of Nuclear Materials 220-222 (1995) 819-822.

[10] K Tokunaga, R P Doerner, R Seraydarian, N Noda, N Yoshida, T Sogabe, T Kato and B Schedler, Modification of tungsten coated carbon by low energy and high flux deuterium irradiation, Journal of Nuclear Materials 307-311 (2002) 126-129.

[11] P Lucasson, M T Robinson, F W Young Jr. (Eds.), Fundamental aspects of radiation damage in metals, USERDA (1975) CONF-751006-P1, p. 42.

[12] F Maury, M Biget, P Vajda, A Lucasson and P Lucasson, Frenkel pair creation and stage I recovery in tungsten crystals irradiated near threshold, Radiation Effects 38 (1978) 53-56.

[13] Q Xu, T Yoshiie and H C Huang, Molecular dynamics simulation of vacancy diffusion in tungsten induced by irradiation, Nuclear Instruments and Methods in Physics Research B 206 (2003) 123-126.

[14] O V Ogorodnikova, B Tyburska, V Kh Alimov and K Ertl, The influence of radiation damage on the plasma-induced deuterium retention in self-implanted tungsten, Journal of Nuclear Materials 415 (2011) S661 - S666.

[15] Standard Practice for Neutron Radiation Damage Simulation by Charge-Particle Irradiation, E521-96, Annual Book of ASTM Standards, Vol. 12.02, American Society for Testing and Materials, Philadelphia, 1996, p. 1.

[16] A W Kleyn, N J Lopes Cardozo, and U Samm, Plasma-Surface Interaction in the context of ITER, Physical Chemistry Chemical Physics 8 (2006), 1761-1774.

[17] H J van der Meiden et al, High sensitivity imaging Thomson Scattering for low temperature plasma, Review of Scientic Instruments 79 (2008) 013505.

[18] D Bohm, The Characteristics of Electrical Discharges in Magnetic Fields ed A Guthrie and R K Wakerling, New York: McGraw-Hill (1949) Chap 3.

[19] V Kh Alimov, M Mayer and J Roth, Differential cross section of the $\mathrm{D}\left({ }^{3} \mathrm{He}, \mathrm{p}\right)^{4} \mathrm{He}$ nuclear reaction and depth profiling of deuterium up to large depths, Nuclear Instruments and Methods in Physics Research B 234 (2005) 169-175.

[20] M Mayer, E Gauthier, K Sugiyama, U van Toussaint, Quantitative depth profiling of deuterium up to large depths, Nuclear Instruments and Methods in Physics Research B 267 (2009) 506512.

[21] M Mayer, SIMNRA User's guide, Report IPP 9/113, Max-Planck-Institut für Plasmaphysik, Garching, 1997.

[22] Fukumoto et al, Deuterium trapping in tungsten damaged by high-energy hydrogen ion irradiation, Journal of Nuclear Materials 390-391 (2009) 572-575.

[23] O V Ogorodnikova, J Roth and M Mayer, Ion-driven deuterium retention in tungsten, Journal of Applied Physics 103 (2008) 034902.

[24] V.Kh. Alimov and J. Roth and M. Mayer, Depth distribution of deuterium in single- and polycrystalline tungsten up to depths of several micrometers, Journal of Nuclear Materials 337339 (2005) $619-623$. 
[25] M. Poon, R.G. Macaulay-Newcombe, J.W. Davis, A.A. Haasz, Effects of background gas impurities during D+ irradiation on D trapping in single crystal tungsten, Journal of Nuclear Materials 337-339 (2005) 629-633.

[26] G.M. Wright and M. Mayer and K. Ertl and G. de Saint-Aubin and J. Rapp, TMAP7 simulations of deuterium trapping in pre-irradiated tungsten exposed to high-flux plasma", Journal of Nuclear Materials 4151 (2011) S636 - S640.

[27] W.M. Shu and E. Wakai and T. Yamanishi, Blister bursting and deuterium bursting release from tungsten exposed to high fluences of high flux and low energy deuterium plasma, Nuclear Fusion 473 (2007) 201.

[28] M. Poon and A.A. Haasz and J.W. Davis, Modelling deuterium release during thermal desorption of $\mathrm{D}^{+}$-irradiated tungsten, Journal of Nuclear Materials 3743 (2008), 390 - 402.

[29] I I Arkhipov, S L Kanashenko, V M Sharapov, R Kh Zalavutdinov and A E Gorodetsky, Deuterium trapping in ion-damaged tungsten single crystal, Journal Nuclear Materials 363-365 (2007) 1168-1172.

[30] M J Norgett, M T Robinson and I M Torrens, A proposed method of calculating displacement dose rates, Nuclear Engineering and Design 33 (1975) 50.

[31] R E Stoller, Point defect survival and clustering fractions obtained from molecular dynamics simulations of high energy cascades, Journal of Nuclear Materials 233-237 (1996) 999-1003.

[32] T Troev, N Nankov and T Yoshiie, Simulation of displacement cascades in tungsten irradiated by fusion neutrons, Nuclear Instruments and Methods in Physics Research B 269 (2011) 566571.

[33] M Biget, R Rizk, P Vajda and A Bessis, On the spontaneous recombination volume of Frenkel defects in irradiated b.c.c. metals, Solid State Communications 16, issue 7 (1975) 949-952.

[34] M Nakawaga, W Mansel, K Böning, P Rosner and G. Vogl, Spontaneous recombination volumes of Frenkel defects in neutron irradiated non-fcc metals, Physical Review B 192 (1979) $742-748$. 